\title{
Multiconfigurational Hartree-Fock close-coupling ansatz: Application to the argon photoionization cross section and delays
}

\author{
T. Carette, ${ }^{1}$ J. M. Dahlström, ${ }^{1}$ L. Argenti, ${ }^{2}$ and E. Lindroth ${ }^{1}$ \\ ${ }^{1}$ Department of Physics, Stockholm University, AlbaNova University Centre, SE-106 91 Stockholm, Sweden \\ ${ }^{2}$ Departamento de Química, Módulo 13, Universidad Autónoma de Madrid, 28049 Madrid, Spain
}

(Received 8 January 2013; published 22 February 2013)

\begin{abstract}
We present a robust, $a b$ initio method for addressing atom-light interactions and apply it to photoionization of argon. We use a close-coupling ansatz constructed on a multiconfigurational Hartree-Fock description of localized states and $B$-spline expansions of the electron radial wave functions. In this implementation, the general many-electron problem can be tackled thanks to the use of the ATSP2K libraries [C. Froese Fischer et al., Comput. Phys. Commun. 176, 559 (2007)]. In the present contribution, we combine this method with exterior complex scaling, thereby allowing for the computation of the complex partial amplitudes that encode the whole dynamics of the photoionization process. The method is validated on the $3 s 3 p^{6} n p$ series of resonances converging to the $3 s$ extraction. Then, it is used for computing the energy dependent differential atomic delay between $3 p$ and $3 s$ photoemission, and agreement is found with the measurements of Guénot et al. [ Phys. Rev. A 85, 053424 (2012)]. The effect of the presence of resonances in the one-photon spectrum on photoionization delay measurements is studied.
\end{abstract}

DOI: 10.1103/PhysRevA.87.023420

PACS number(s): 32.80.Aa, 31.15.A-, 32.80.Zb

\section{INTRODUCTION}

Since its discovery, the photoelectric effect has occupied a forefront position among the processes triggered by the interaction between matter and radiation. This is due to the relevance of photoionization for a range of naturally occurring phenomena, as for example those determining the opacity of astrophysical objects [1], or leading to radiation damage of biological systems [2], as well as for many technological applications. A quantitative description of photoionization is required to understand and control these processes. Research in this direction has a long history [3,4] and generated vast literature. The most recent development in this area includes the advent of several new light sources providing shorter pulses, higher intensities, or shorter wavelengths [5,6]. During recent years, such sources have been utilized to also gain knowledge of the temporal aspects of photoionization $[7,8]$.

A reliable description of the photoionization event requires a good representation of the atomic or molecular system during all the stages of its interaction with light. This includes the structure of the initial bound state, as well as of the final parent or target states, but also the coupling between the released photoelectron and the relaxing parent ion. In this work, we present a tool that permits one to treat many-body effects in bound and continuum states of many-electron atoms, irrespective of how the photoionization process is to be treated. Depending on the intensity, pulse length, and monochromaticity of the light the appropriate method may be found everywhere on the range from the standard one-photon time-independent formalism, to the explicit solution of the time-dependent Schrödinger equation. Since the latter approach quickly grows computationally heavy for all but the smallest systems, it is usually necessary to stick to a simplified treatment of correlation for

*eva.lindroth@fysik.su.se larger systems. The procedure we outline here allows for a systematic refinement of many-body effects and the unified approach for time-independent and time-dependent calculations permits further tests and verifications of the amount of correlation in the latter by comparison with the effect on the former.

In the present study we validate the approach and verify that the underlying approximations are justified by comparing photoabsorption cross sections for the argon atom, computed in the weak-field limit, with existing experimental and theoretical data. Furthermore, we show that the delay in the photoelectron ejection observed experimentally [8,9] can be largely explained by the single photoionization scattering phases when corrected for the phase shift introduced in the experiment by the probe photon [10].

For the description of the wave functions in the continuum two different approaches dominate the literature. One possibility is to match a numerical solution, computed inside an inner spherical region, with the appropriate asymptotic solution in the outer region, whose analytical form is known. This is the basis of most scattering methods such as, e.g., the $R$-matrix method, originally adapted for the photoionization problem by Burke and Taylor [11] and recently presented also in a time-dependent version [12], as well as the $K$-matrix method $[13,14]$. An alternative solution is to use complex scaling, where radial coordinates are rotated in the complex plane. In this case, the photoabsorption cross section can be directly computed as the imaginary part of the frequency-dependent polarizability [15-17]. Complex scaling methods present some advantages as well as some disadvantages with respect to scattering methods. On the one hand, with complex scaling, resonance states have a more transparent representation since they appear directly as eigenstates of the complex-scaled Hamiltonian with a negative imaginary part of the energy. On the other hand, the complex-scaled wave functions differ from the generalized eigenstates of the unscaled Hamiltonian. Therefore, properties that can only be extracted from the 
ionized part of the wave function are not easily obtainable. This problem is circumvented using exterior complex scaling [18], where the radial coordinate is scaled only outside a sufficiently large region. In this method, the wave function in the inner unscaled region can be directly analyzed. Exterior complex scaling has been extensively applied to electron and photon scattering problems [19-22] (see [23] for a review).

The description of the target and parent-ion states is an atomic structure problem than can be treated with the tools targeting correlation in bound states, e.g., many-body perturbation theory, coupled cluster, configuration interaction, etc. (see, e.g., [24-26]). Most of these approaches can subsequently be combined with different representations of the continuum. Configuration interaction, for example, can be used to either represent the atomic system in the inner $R$-matrix region or be employed together with complex scaling. A method that is particularly convenient when aiming at problems involving single atomic continua, but nontrivial configurations, is the multiconfiguration Hartree-Fock (MCHF) method [25,27]. It is known to yield accurate correlation models for general bound states [28] while still limiting the number of configurations required for convergence. MCHF has often been used in combination with the $R$-matrix method (see, e.g., Ref. [29]), but also together with other approaches to describe the continuum (see, e.g., Ref. [30]). Here, we employ the close-coupling ansatz, originally introduced by Massey and Mohr [31,32], for the atomic wave function. The localized part of the wave function is expressed in terms of MCHF configurations; our implementation is based on the atomicstructure package ATSP $2 \mathrm{~K}$ [33], while the radial component that corresponds to the photoelectron is expanded on an extensive $B$-spline basis. We use exterior complex scaling for an explicit description of resonances and a good representation of the continuum.

This paper is organized as follows. In Sec. II we outline the present implementation of the close-coupling ansatz, and in Sec. III the calculation of photoionization parameters is discussed. To validate the method, we compare our results for the photoionization of the argon atom with other theoretical and experimental data in Sec. IV. Argon provides a good benchmark because it is comparatively light, so a nonrelativistic description is appropriate, and its resonance profiles and angular distribution, which encode the full information about the complex partial amplitudes [34], have been the target of many experimental and theoretical studies [11,29,35-41]. Figure 1 is a diagram of the levels of neutral argon accessible through one-photon transition, shown on an energy scale corresponding to photoionization from the ground state. Above the $3 s 3 p^{6}$ threshold, we only mark the levels of $\mathrm{Ar}^{+}$in black and gray. In Sec. IV, we focus our attention on the photon energy range between 26 and $30 \mathrm{eV}$ which comprises the $3 s 3 p^{6} n p$ autoionizing series, which entails strong configuration interaction. In Sec. V we use our approach to estimate the one-photon Wigner-like delay in the experimentally relevant region from 32 to $42 \mathrm{eV}$. The importance of the presence of many resonances affecting the atomic delay is demonstrated. Finally, in Sec. VI we draw our conclusions.

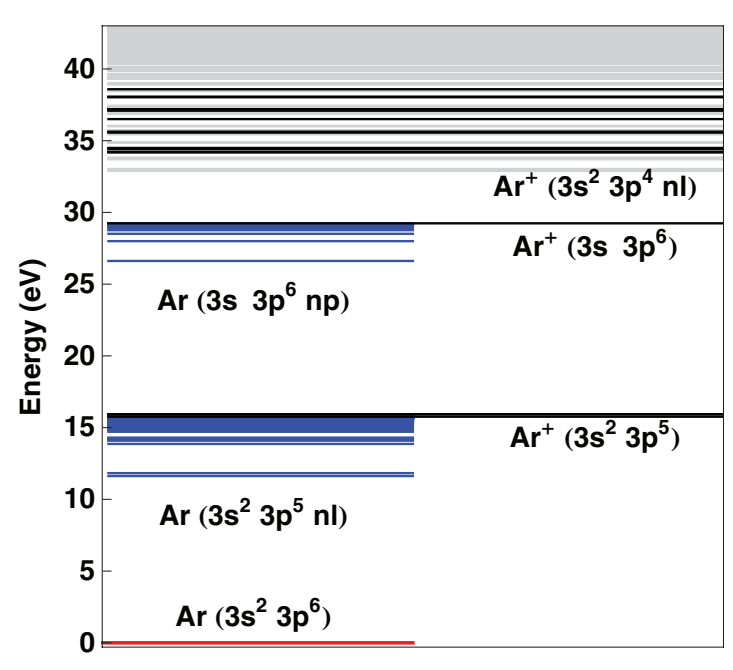

FIG. 1. (Color online) Diagram of the neutral argon levels and ionization thresholds accessible through one-photon transitions. Data are taken from the NIST atomic database [42]. The red level is the Ar ground state, and the blue states are singly excited bound and autoionizing states. Black lines are ionization thresholds that are included in our analysis. Gray lines are other ionization thresholds which are neglected in the present paper (cf. the Appendix).

\section{THE CLOSE-COUPLING ANSATZ}

\section{A. Exterior complex scaling}

In our implementation of the close-coupling ansatz, the states in the continuum are evaluated on an exterior complexscaled (ECS) radial coordinate

$$
r \rightarrow r^{(\theta)} \equiv \begin{cases}r & \text { if } r<R_{0} \\ R_{0}+e^{i \theta}\left(r-R_{0}\right) & \text { if } r \geqslant R_{0} .\end{cases}
$$

As mentioned earlier, this approach provides a good description of the photoabsorption cross section in general, and of transiently bound states, in particular. In the present context, ECS has two further advantages. First, as detailed in Sec. II B, we employ existing MCHF codes [33] to describe the bound states. If $R_{0}$ is large enough, the output of these programs can be directly used in conjunction with additional complex-scaled functions without any modification. In fact, this was the reason why Simon [18], who wanted to treat molecules without having to scale the molecular potential, introduced ECS to begin with. Second, with ECS we are able to analyze the ionized part of the wave function to determine partial complex amplitudes. This will be discussed in more detail in Sec. III.

\section{B. Multiconfiguration Hartree-Fock method}

In the MCHF method, the atomic wave function is expanded on an orthonormal set of symmetry adapted linear combinations of Slater determinants [25,27], $\left\{\Phi\left(\gamma_{i} L S\right)\right\}$, known as configuration state functions (CSF),

$$
\Psi=\sum_{j} c_{j} \Phi\left(\gamma_{j} L S\right) .
$$

The radial functions $\left\{P_{n l}(r)\right\}$, which define the occupied and correlation spin orbitals, and the $\left\{c_{j}\right\}$ mixing coefficients are 
then optimized to minimize the energy functional

$$
E\left[\left\{P_{n l}(r)\right\},\left\{c_{j}\right\}\right]=\langle\Psi|H| \Psi\rangle,
$$

where $H$ is the nonrelativistic atomic Hamiltonian with infinite mass nucleus, under the orthogonality constraint for the radial orbitals $\left\{P_{n l}(r)\right\}$ :

$$
\int P_{n l}(r) P_{n^{\prime} l}(r) d r=\delta_{n n^{\prime}}
$$

We use the ATSP2K package [33] to solve the MCHF problem.

The close-coupling expansion is defined by a set of $N$ electron target states $\left(\Psi_{a}\right)$ coupled to an extensive set of oneparticle states with defined orbital angular momentum $l$. This group of $(N+1)$-particle states is further complemented by a set of localized states $\left(\chi_{\xi}\right)$. The wave function of the atomic state before or after photoabsorption can be expressed as

$$
\Psi=\sum_{\xi} c_{\xi} \chi_{\xi}+\sum_{\alpha, n} c_{\alpha n} \mathcal{A}_{L S}\left\{\Psi_{a} P_{n l}\left(r_{N+1}\right)\right\}
$$

where $\alpha$ is the channel index, indicating target state $a$ and photoelectron angular momentum $l$, and $\mathcal{A}_{L S}$ yields a symmetry adapted, antisymmetric $(N+1)$-electron wave function. The $N$-electron CSF are built from the orbitals obtained in the MCHF calculation, and we find the target states $\left(\Psi_{a}\right)$ as the linear combinations that diagonalize the total $N$-electron Hamiltonian. We further use these orbitals to set up the $(N+1)$-electron CSF that form the $\chi_{\xi}$. All intermediate angular couplings compatible with the total symmetry under examination are included in the close-coupling expansion which, in this sense, is therefore complete. As in MCHF-based $R$-matrix approaches [29], orbitals that are occupied in the target states, and which derive from a MCHF optimization, are kept frozen throughout the calculations. A discretized description of the continuum orbitals $P_{n l}(r)$ describing the photoelectron is assumed. The configuration interaction coefficients $c_{\xi}$ and $c_{\alpha n}$ in Eq. (5) give the close-coupling ansatz a great flexibility. Even if occupied orbitals are optimized on the parent states, the second term of Eq. (5) is able to account for the orbital adjustment between the $N$ - and $N+1$ electron systems. One drawback of this approach is that highly excited configurations or configurations involving correlation orbitals often appear as discrete states that are embedded in the continua, slightly above the physical states described by the model. These states appear as artifacts of the calculation in the photoionization cross section, so-called pseudoresonances.

\section{Description of the photoelectron}

The photoelectron radial function basis $\left\{P_{n l}\right\}$ [cf. Eq. (5)] is expanded in $B$-splines [43],

$$
P_{n l}\left(r^{(\theta)}\right)=\sum_{i} c_{i} B_{i}^{k}\left(r^{(\theta)}\right) .
$$

The $B$-splines of order $k$ are defined as piecewise polynomials of order $k-1$ between predefined isolated points, called knots. In this work, we use $k=7$. With exterior complex scaling, it is convenient to let the knot sequence follow the scaled $r$ in Eq. (1), i.e., $r^{(\theta)}$ [44]. $B$-splines can be used to form effectively complete basis sets for the description of localized electron partial wave packets, in particular in atomic problems [45,46]. Since $B$-splines are nonorthogonal, however, they cannot be directly used in combination with ATSP2K. The expansion coefficients in Eq. (6) are obtained through the following procedure. First the MCHF orbitals, computed on a radial grid using the MCHF program of ATSP $2 \mathrm{~K}$, are fitted with $B$-splines using the $\mathrm{W} \_$BSW program of the $B$-spline $R$-matrix (BSR) package [47]. The exterior complex scaling radius $R_{0}$ is chosen to be larger than the radius at which the MCHF orbitals numerically vanish, therefore, the MCHF orbitals are expressed in the region where $r$ is unscaled. Second, for each orbital obtained at the MCHF stage, the $B$-spline corresponding to the largest mixing coefficient in $P_{n l}(r)$ is removed. Finally, the remaining $B$-splines are orthogonalized to the MCHF orbitals with the Gram-Schmidt algorithm. When combined, the occupied $P_{n l}(r)$ and the orthogonalized $B$-spline set form an orthonormal basis equivalent to the original $B$-spline radial basis. The $B$-splines which do not vanish at $R_{0}$ are complex [23]; as a consequence, the final basis set is complex as well.

\section{ATOMIC PHOTOIONIZATION USING EXTERIOR COMPLEX SCALING}

The correlated many-electron basis set that we use to describe the system before and after photoabsorption is obtained by diagonalizing the full complex-scaled Hamiltonian $H^{\theta}$ projected on the space spanned by the ansatz in Eq. (5). The resulting eigenenergies $E_{k}^{\theta}$, corresponding to the eigenvectors $\left|k^{\theta}\right\rangle$, are in general complex. Those corresponding to bound states have a vanishing imaginary part of the energy; some complex eigenvalues, which correspond to resonances, are largely independent of the rotation angle; finally, the remaining eigenvalues correspond to the unstructured continuum.

\section{A. The total cross section}

The single-photon absorption cross section can be expressed in terms of the dynamic polarizability of the ground state $|0\rangle$ of the atom,

$$
\sigma(\omega)=-\frac{e^{2}}{4 \pi \epsilon_{0}} \frac{4 \pi}{3} \frac{\omega}{c} \operatorname{Im}\left\langle 0\left|(\hat{\varepsilon} \cdot \mathbf{R}) G_{0}^{+}\left(E_{0}+\hbar \omega\right)(\hat{\varepsilon} \cdot \mathbf{R})\right| 0\right\rangle,
$$

where $\mathbf{R}=\sum_{j} \mathbf{r}_{j}, G_{0}^{+}(E)=\left(E-H+i 0^{+}\right)^{-1}$ is the Green's function of the field-free Hamiltonian, $\hbar \omega$ is the photon energy, and $\hat{\varepsilon}$ is the direction of the laser polarization.

With complex scaling, the Green function is constructed as a sum over the many-electron basis set components as [15,16]

$$
\sigma(\omega)=-\frac{e^{2}}{4 \pi \epsilon_{0}} \frac{4 \pi}{3} \frac{\omega}{c} \operatorname{Im}\left[\sum_{k} \frac{\left\langle 0\left|\hat{\varepsilon} \cdot \mathbf{R}^{(\theta)}\right| k^{\theta}\right\rangle\left\langle k^{\theta}\left|\hat{\varepsilon} \cdot \mathbf{R}^{(\theta)}\right| 0\right\rangle}{E_{0}-E_{k}^{\theta}+\hbar \omega}\right],
$$

where $\left|k^{\theta}\right\rangle$ and $\left\langle k^{\theta}\right|$ are right and left eigenvectors of the complex-scaled Hamiltonian $H^{\theta}$, with complex eigenvalues $E_{k}^{\theta}$. For the field-free Hamiltonian $H^{\theta}$, the left eigenvectors are simply the transpose of the right eigenvectors (see, e.g., [48]). The sum over $\left|k^{\theta}\right\rangle$ goes over all eigenstates to $H^{\theta}$ including both resonances and continuum states. We note that since 
resonances appear directly as eigenstates of $H^{\theta}$ the effect of a resonance on the photoionization process is accordingly accounted for by a single term in Eq. (7).

\section{B. The Fano profile}

As was shown by Fano [49] the cross section in the vicinity of an isolated resonance, situated at $E_{r}$ (relative to $E_{0}$ ) and with autoionization width $\Gamma$, can be parametrized as

$$
\sigma(\epsilon)=\frac{(q+\epsilon)^{2}}{1+\epsilon^{2}} \sigma_{a}+\sigma_{b}
$$

where $q$ is the so-called asymmetry parameter and $\epsilon$ is defined as

$$
\epsilon=\frac{\hbar \omega-E_{r}}{\Gamma / 2},
$$

while $\sigma_{a}$ and $\sigma_{b}$ are slowly varying as a function of $\epsilon$.

Looking at the contribution from a specific resonance in the sum on the right-hand side of Eq. (8), we can indeed put each such contribution in the form of Eq. (9). The derivation is detailed in Ref. [16]; here we just give the results. Each resonance $k$ contributes with

$$
\sigma_{k}\left(\epsilon_{k}\right)=\frac{\left(q_{k}+\epsilon_{k}\right)^{2}}{1+\epsilon^{2}} \sigma_{a}^{k}+\sigma_{\min }^{k},
$$

where the reduced energy $\epsilon_{k}$ of the resonance $|k\rangle$ is defined as in Eq. (10), with $E_{k}^{r}=\operatorname{Re}\left[E_{k}\right]-E_{0}$ being the position of the resonance, and $\Gamma_{k}=-2 \operatorname{Im}\left[E_{k}\right]$. The asymmetry parameter $q_{k}$, but also $\sigma_{a}^{k}$ and $\sigma_{\min }^{k}$, are here constants and are determined by the resonance parameters and by the complex dipole matrix element between the ground state and the considered resonant state, the numerator in the term in brackets in Eq. (8). Labeling it $R_{k}+i I_{k}=\left\langle k^{\theta}\left|\hat{\varepsilon} \cdot \mathbf{R}^{(\theta)}\right| 0\right\rangle^{2}[16]$, the three constants are found to be

$$
\begin{gathered}
q_{k}=b_{k}-\frac{I_{k}}{\left|I_{k}\right|} \sqrt{b_{k}^{2}+1}, \\
\sigma_{a}^{k}=\frac{e^{2}}{4 \pi \epsilon_{0}} \frac{4 \pi}{3} \frac{1}{\hbar c}\left(\frac{R_{k}-2 I_{k} E_{k}^{r} / \Gamma_{k}}{2 q_{k}}\right), \\
\sigma_{\min }^{k}=-\frac{e^{2}}{4 \pi \epsilon_{0}} \frac{4 \pi}{3} \frac{1}{\hbar c}\left(\frac{2 R_{k} E_{k}^{r} / \Gamma_{k}+q_{k}^{2} I_{k}}{2 q_{k} b_{k}}\right),
\end{gathered}
$$

where

$$
b_{k}=\frac{I_{k} \Gamma_{k}+2 E_{k}^{r} R_{k}}{R_{k} \Gamma_{k}-2 E_{k}^{r} I_{k}} .
$$

If the sum over all the other contributions in Eq. (8), from other resonances as well as from the smooth continuum, just give slowly varying contributions in the vicinity of the resonance $k$, then it is clear that the total cross section is well described by Eq. (9). This condition should be fulfilled if the resonance is far from other resonances and from thresholds.

The resonance parameters $E_{r}, \Gamma$, and $q$ in Eq. (12) are often obtained from experiment through a fit to a Fano profile. For validation purposes, we compare these with our calculated values in Sec. IV. Another parameter that is also obtainable from experiments is the coefficient $\rho^{2}$ [50],

$$
\rho^{2}=\frac{\sigma_{a}}{\sigma_{a}+\sigma_{b}} .
$$

Here $\sigma_{a}$ can be calculated directly from Eq. (13), while $\sigma_{b}$ is not given by $\sigma_{\min }$ alone but from the full sum over states $k$ in Eq. (8). However, in the limit that Eq. (9) is valid, $\sigma_{b}$ can be obtained from Eq. (8) at $\epsilon=-q$.

\section{Partial cross sections}

Equation (8) gives a convenient way to obtain the total cross section. For the extraction of the partial cross sections we analyze the many-electron, one-photon perturbed wave function $\Xi\left(\omega, \mathbf{r}_{1}, \mathbf{r}_{2}, \ldots, \mathbf{r}_{N}\right)$, which is a function of the photon energy as well as of the coordinates of all the particles in the system. The perturbed wave function solves the following inhomogeneous differential equation:

$$
\begin{gathered}
\left(H-E_{0}-\hbar \omega\right) \Xi\left(\omega, \mathbf{r}_{1}, \mathbf{r}_{2}, \ldots, \mathbf{r}_{N+1}\right) \\
\quad=-(\hat{\varepsilon} \cdot \mathbf{R}) \Psi_{0}\left(\mathbf{r}_{1}, \mathbf{r}_{2}, \ldots, \mathbf{r}_{N+1}\right),
\end{gathered}
$$

where the source term on the right-hand side describes the excitation from the ground state by the dipole operator acting on all electrons. The concept of perturbed wave functions was introduced by Sternheimer [51], and it remains a useful tool also today, with novel applications emerging in the field of attosecond science [10]. Here it is obtained through direct summation over our many-body basis as

$$
\left|\Xi^{\theta}\right\rangle=\sum_{k}\left|k^{\theta}\right\rangle \frac{\left\langle k^{\theta}\left|\hat{\varepsilon} \cdot \mathbf{R}^{\theta}\right| 0\right\rangle}{E_{0}-E_{k}^{\theta}+\hbar \omega},
$$

which represents the full many-electron wave packet formed by absorption of one photon from the initial state. To extract partial physical observables, $\Xi^{\theta}$ is projected on a specific channel, $\alpha$, defined by a bound $N$-electron state of the parent ion and a fixed angular momentum of the outgoing electron. As a result, we obtain a radial perturbed wave function (PWF), denoted $\rho_{\alpha}^{\theta}(\omega, r)$, which is a superposition of the radial functions $P_{n l}(r)$ introduced in Eqs. (5) and (6). Figure 2 shows the complex and imaginary parts of $\rho_{\alpha}^{\theta}(\omega, r)$. For $r<R_{0}, \rho_{\alpha}^{\theta}(\omega, r)$ is equal to the radial perturbed wave function without complex scaling, while in the complex-scaled region $r>R_{0}$ it is artificially damped. As a second step, the complex amplitude $A_{\alpha}(\omega)$ of the PWF is obtained by fitting $\rho_{\alpha}(\omega, r)$ to a linear combination of the energy-normalized regular $F_{l}^{E}$ and irregular $G_{l}^{E}$ Coulomb functions

$$
\begin{aligned}
\operatorname{Re}\left[\rho_{\alpha}(\omega, r)\right]= & \operatorname{Im}\left[A_{\alpha}(\omega)\right] F_{l}^{E}(-1 / k, k r) \\
& -\operatorname{Re}\left[A_{\alpha}(\omega)\right] G_{l}^{E}(-1 / k, k r),
\end{aligned}
$$

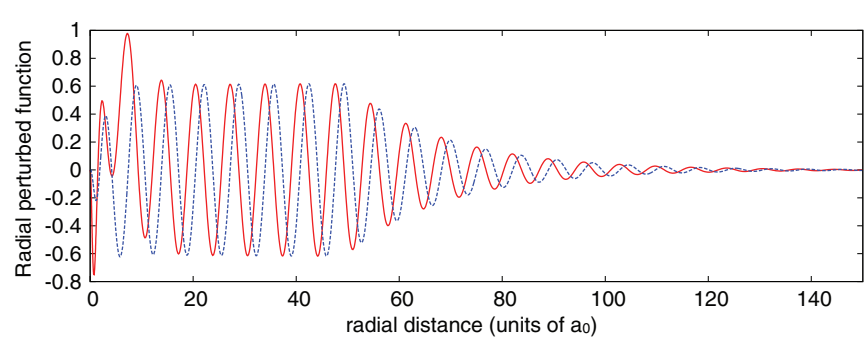

FIG. 2. (Color online) Radial perturbed function for the $3 p \rightarrow \epsilon d$ channel, in length gauge, for $\epsilon=-q$ in the vicinity of the $3 s 3 p^{6} 4 p$ resonance. The red and dashed blue curves are its real and imaginary parts, respectively. 
where here, $k$ is the photoelectron wave number and $E$ is the corresponding energy. The fit is carried out for a radial distance $r<R_{0}$ so that it is not affected by the complex scaling, but large enough that the asymptotic behavior is established. In practice, we compute $\Xi^{\theta}$, and $A_{\alpha}$, for a specific $M$ magnetic quantum number. In the following, however, observables extracted from the complex amplitudes are implicitly averaged over orientations except if otherwise stated. The absolute square of the amplitudes is related to the electron flux and, hence, to the partial cross section, as

$$
\sigma_{\alpha}(\omega)=\frac{4 \pi}{3} \frac{e^{2}}{\left(4 \pi \varepsilon_{0}\right) \hbar c} a_{0}^{2} \frac{\hbar \omega}{\pi}\left|A_{\alpha}(\omega)\right|^{2} .
$$

Note that the amplitude (and the cross section) is that of a photoelectron in channel $\alpha$. The spin-averaged, angular dependent photoelectron amplitude, with given $M$ magnetic quantum number, is obtained after multiplying it by spherical harmonics coupled by Clebsch-Gordan coefficients as

$$
A_{\alpha, M}(\omega) \sum_{m} Y_{l m}(\theta, \varphi)\left\langle L_{a}(M-m) ; \operatorname{lm} \mid L_{\alpha} M\right\rangle,
$$

where $L_{a}$ is the angular momentum of the $N$-electron system $\Psi_{a}$, and $L_{\alpha}$ that of the total $(N+1)$ system. Expression (21) can be used to obtain the angular dependence of photoelectrons ionized by polarized light

$$
\frac{d \sigma(\omega)}{d \Omega}=\frac{\sigma(\omega)}{4 \pi}\left[1+\beta(\omega) P_{2}(\cos \phi)\right]
$$

where $P_{2}$ is the second-order Legendre polynomial and $\phi$ is the angle between the polarization direction and the direction of the photoelectron. The parameter $\beta(\omega)$ is

$\beta(\omega)=\left(\sum_{\alpha \beta} \Theta(\alpha ; \beta) \operatorname{Re}\left[A_{\alpha}^{*}(\omega) A_{\beta}(\omega)\right]\right)\left(\sum_{\alpha}\left|A_{\alpha}(\omega)\right|^{2}\right)^{-1}$,

where $\Theta(\alpha ; \beta)$ is a purely angular factor given by

$$
\begin{aligned}
\Theta(\alpha ; \beta)= & (-1)^{L_{0}-L_{a}+l_{\alpha}+l_{\beta}+1} i^{-l_{\alpha}+l_{\beta}} \delta(a, b) \\
& \times \sqrt{\frac{2\left(2 l_{\alpha}+1\right)\left(2 l_{\beta}+1\right)\left(2 L_{\alpha}+1\right)\left(2 L_{\beta}+1\right)}{3}} \\
& \times\left\langle l_{\alpha} 0 l_{\beta} 0 \mid 20\right\rangle\left\{\begin{array}{lll}
l_{\alpha} & l_{\beta} & 2 \\
L_{\beta} & L_{\alpha} & L_{a}
\end{array}\right\}\left\{\begin{array}{ccc}
1 & L_{\alpha} & L_{0} \\
L_{\beta} & 1 & 2
\end{array}\right\},
\end{aligned}
$$

where the index 0 refers to the initial state, and $\alpha=\left(a, l_{\alpha}\right)$ and $\beta=\left(b, l_{\beta}\right)$ refer to the two interfering channels.

\section{Extraction of resonance properties from the partial cross section}

Properties like $\rho^{2}$, defined in Eq. (16), can be extracted from the partial channels as well as from the total cross section. For this we follow Kabachnik and Sazhina [34] and Sorensen et al. [36], and express the amplitude of the PWF as the combination of a resonant $A_{\alpha}^{R}$ and a decoupled $A_{\alpha}^{D}$ complex amplitude:

$$
\begin{gathered}
A_{\alpha}(\epsilon)=\mathcal{F}(\epsilon) A_{\alpha}^{R}+A_{\alpha}^{D}(\epsilon), \\
\mathcal{F}(\epsilon)=\frac{q+\epsilon}{i+\epsilon}=(\epsilon-i) \frac{q+\epsilon}{1+\epsilon^{2}},
\end{gathered}
$$

where $\epsilon$ is defined as in Eq. (10). If the decay of the bound component of the resonance is treated to lowest order in the Coulomb interaction, these amplitudes have the same phase up to a $\pi$ shift $[34,50]$. We show in Sec. IV C that it remains valid in the case of the $3 s 3 p^{6} n p$ resonances of Ar, and that the effect of the higher-order contributions, present in our approach, is small. From $A_{\alpha}^{R}$ and $A_{\alpha}^{D}(\epsilon)$, we can calculate $\rho^{2}$ [50] as

$\rho^{2}=\frac{\sigma_{a}}{\sigma_{a}+\sigma_{b}}=1-\frac{\sigma_{b}}{\sigma_{a}+\sigma_{b}}=1-\sum_{\alpha} \frac{\left|A_{\alpha}^{D}\right|^{2}}{\left|A_{\alpha}^{R}+A_{\alpha}^{D}\right|^{2}}$,

where we have used the fact that the nonresonant background cross section $\sigma_{b}$ is obtained from the sum of the squares of the decoupled amplitudes $A_{\alpha}^{D}$. For the extraction of $A_{\alpha}^{R}$ and $A_{\alpha}^{D}(\epsilon)$, we suppose first that $A_{\alpha}^{R}$ can be considered a constant. Provided $A_{\alpha}^{D}(\epsilon)$ is slowly varying, we are able to describe it around a certain $\epsilon$ as an $n$-term Taylor expansion. Then $A_{\alpha}^{R}$ can be obtained from the $n$th derivative of Eq. (25) as

$$
A_{\alpha}^{R}=\frac{d^{n} A_{\alpha}(\epsilon)}{d \epsilon^{n}}\left(\frac{d^{n} \mathcal{F}(\epsilon)}{d \epsilon^{n}}\right)^{-1} .
$$

The number of terms $n$ used is decided from the convergence of $A_{\alpha}^{R}$ for a given $\epsilon$, and the stability of $A_{\alpha}^{R}$ when $\epsilon$ is varied can be used for assessing the reliability of the procedure. Making a comparison between the values obtained for $\rho^{2}$ from the total cross section Eq. (16), and from the partial amplitudes, the right-hand side of Eq. (27), is a test of the extraction of the partial cross sections as well as of the Fano parametrization itself.

\section{APPLICATION TO ARGON $3 s \rightarrow n p$ RESONANCE REGION}

\section{A. Details of the calculations}

In the present section, we briefly outline the construction of the MCHF model. Our approach is similar to those followed by Berrah et al. [29] and by Burke and Taylor [11]; the reader can find further details in the original references. The spectroscopic orbitals $\{1 s, 2 s, 2 p, 3 s, 3 p\}$ are optimized by performing a Hartree-Fock (HF) calculation on the $3 p^{5}$ ${ }^{2} P^{o}$ state of $\mathrm{Ar}^{+}$. Then, the $\{\overline{3 d}, \overline{4 s}, \overline{4 p}\}$ correlation orbitals are optimized in a MCHF calculation on the lowest ${ }^{2} S$ state of $\mathrm{Ar}^{+}$including single excitations of the $3 s 3 p^{6}$ configuration, as well as the $3 s 3 p^{4} \overline{3 d}^{2}$ complex. As illustrated in Table I, this state is characterized by a strong configuration mixing.

TABLE I. List of mixing coefficients in the MCHF expansion of the lowest ${ }^{2} S\left(3 s 3 p^{6}\right)$ state of $\mathrm{Ar}^{+}$.

\begin{tabular}{lr}
\hline \hline $\mathrm{CSF}$ & Mixing coefficient \\
\hline $3 s 3 p^{6}$ & 0.83498723 \\
$3 s^{2} 3 p^{4}\left({ }^{1} D\right) 3 d$ & -0.52070520 \\
$3 s 3 p^{4}\left({ }^{1} S\right) 3 d^{2}\left({ }^{1} S\right)$ & 0.09817089 \\
$3 s 3 p^{4}\left({ }^{3} P\right)\left[{ }^{4} P\right] 3 d^{2}\left({ }^{3} P\right)$ & -0.09655511 \\
$3 s 3 p^{4}\left({ }^{1} D\right) 3 d^{2}\left({ }^{1} D\right)$ & -0.08964443 \\
$3 s 3 p^{5}\left[{ }^{1} P\right] 4 p$ & -0.05536407 \\
$3 s^{2} 3 p^{4}\left({ }^{1} S\right) 4 s$ & -0.03427725 \\
$3 s 3 p^{4}\left({ }^{3} P\right)\left[{ }^{2} P\right] 3 d^{2}\left({ }^{3} P\right)$ & -0.01888098 \\
$3 p^{6} 4 s$ & 0.00827551 \\
$3 s 3 p^{5}\left[{ }^{3} P\right] 4 p$ & -0.00088679 \\
\hline \hline
\end{tabular}


The set of CSF included in the diagonalization of the $N$ electron Hamiltonian defining the ${ }^{2} S$ parent states entering the close-coupling expansion [see Eq. (5)], is the same as the one used in the MCHF calculation. For the ${ }^{2} P^{o}$ parents, we include the single excitations of the $3 s^{2} 3 p^{5}$, and the doubly excited CSF arising from the $3 s^{2} 3 p^{3} \overline{3 d}^{2}, 3 p^{5} \overline{3 d}^{2}$, and $3 p^{6} \overline{4 p}$ configurations.

This model yields a threshold energy for the $3 s$ photoionization of argon of $29.73 \mathrm{eV}$, compared to the experimental value of $29.24 \mathrm{eV}$ [52]. The difference of $0.49 \mathrm{eV}$ between theory and experiment is due to the lack of balance between the description of the ion and of the ground state of the neutral atom. Since this discrepancy is mostly reflected in a collective shift of all spectral features, we simply present all our results on a photon energy scale shifted downward by $0.49 \mathrm{eV}$ to compare with the experiment, rather than using any semiempirical corrections.

The set of localized configuration state functions $\chi_{\xi}$ in Eq. (5) comprises all single and double excitations from $3 s^{2} 3 p^{6}$, all triple excitations with at least two $\overline{3 d}$ electrons, and the $3 s^{2} 3 p^{2} \overline{3 d}^{3} \overline{4 p}$ configuration, i.e., all those listed in Table II of Ref. [29].

The final close-coupling expansion using $B$-splines is obtained as explained in Secs. II B and II C. For small radii, a dense $B$-spline basis is necessary to correctly describe the inner orbitals. A $0.1 / Z a_{0}$ step size is used from the origin to $1 / Z a_{0}$. Then, the knot spacing increases exponentially up to $0.9 a_{0}$. The knot sequence is then linear up to $80 a_{0}$. It is generated by the W_BSW program of the BSR package [47]. To represent high- $n$ Rydberg states, we extend the node set with an exponential sequence of 100 nodes from 80 to $R=400 a_{0}$. The final basis contains $249 B$-splines. We ascertain that the results are stable with respect to changes of the complex scaling parameters by varying $R_{0}$, the radial distance at which exterior complex scaling begins, from 50 to $80 a_{0}$, and by doubling the complex scaling angle $\theta$ in Eq. (1).

\section{B. Total photoionization cross-section and Fano profile parameters}

The upper panel of Fig. 3 shows part of the complex rotated energy spectrum for the ${ }^{1} P^{o}$ manifold, obtained from the diagonalization of the field-free Hamiltonian $H^{\theta}$ using two different complex rotation angles. As expected, the complex resonance energies are virtually independent of the rotation angle. For example, all the parameters of the $3 s 3 p^{6} n p$ resonances agree to one ppm up to the $n=13$ state. The pseudocontinuum energies, instead, follow a $\theta$-dependent path in the complex plane [48]. Also Rydberg states with $n>13$, that do not fit in the computational box, show a $\theta$ dependence.

The lower panel of Fig. 3 shows total cross sections obtained in length and velocity gauge. The thick lines correspond to the cross sections obtained by including the complete sum over the basis set in Eq. (8), while the thin lines are computed including only the $3 s 3 p^{6} n p$ resonance series by summation over the corresponding $\sigma_{k}$ given in Eq. (11). The latter curves reproduce well the resonant structure of the cross section, but the remaining nonresonant contributions are required to obtain the absolute value of the cross section. The good agreement of the calculated total cross section in the two gauges in

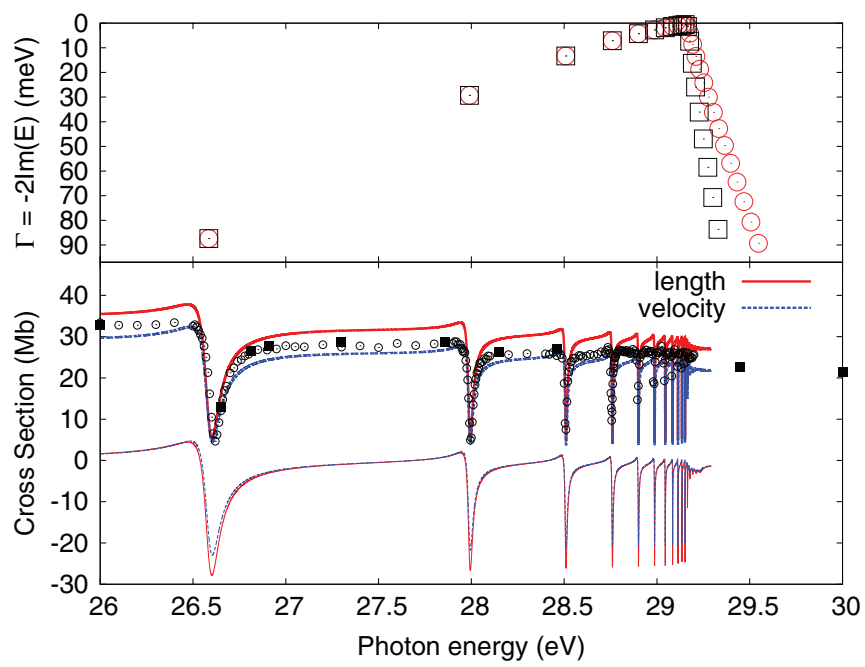

FIG. 3. (Color online) Top panel: Details of the complex energies of the field-free Hamiltonian of argon calculated for the ${ }^{1} P^{o}$ symmetry with $\theta=0.055 \mathrm{rad}$ (red circles) and $0.11 \mathrm{rad}$ (black squares). Bottom panel: Comparison of the total calculated cross sections (thick lines), in length (full red curve) and velocity (dashed blue curve) gauges. The empty circles are the experimental values of Berrah et al. [29], in arbitrary units, scaled as in the original paper. The black squares are absolute cross-section measurements from Samson and Stolte [53]. The thin lines are the cross sections computed by including only the $3 s 3 p^{6} n p$ resonances in the basis set.

the transmission window suggests that $\sigma_{b}$ in Eq. (9) is well described and that the discrepancy comes mostly from $\sigma_{a}$.

Table II lists the parameters for some of the $3 s 3 p^{6} n p$ autoionizing states computed with our method, in length and velocity gauges, and compares them to Refs. [29,38]. Our results are generally consistent with those obtained with the $R$-matrix method [29] with similar choices for the computational model and basis. The widths are slightly larger than the experimental ones and those obtained from the fits to the $R$-matrix calculation. The agreement of the $q$ parameter from the $R$-matrix calculation is good, but both calculations show rather large deviations from the experiment. Reference [29] attributes this discrepancy to the difficulty to accurately represent the nonresonant background in the close-coupling expansion. The marked gauge dependence of $\sigma_{a}$ discussed in the previous paragraph supports the fact that the contribution from the resonant channel is not yet converged.

\section{Partial cross sections and angular distribution}

As a test of the robustness of the method outlined in Sec. III C, we compare the total cross section computed with Eq. (7) to the sum over the partial amplitudes of the $3 p$ photoemission channels computed with Eq. (20). This is shown for the length gauge in Fig. 4. As can be seen in the lower panel, the relative error between the two approaches is of the order of $10 \mathrm{ppm}$ at most energies, which is close to our computational accuracy. For the high $n$ resonances, spurious long-range effects affect the channel perturbed radial functions. Therefore, their matching to Coulomb waves [see Eq. (19)] is less accurate at the considered $R_{0}$, but the error remains consistently of the order of $0.1 \%$. Above the $3 s$ ionization threshold, the $3 s 3 p^{6} \epsilon p$ 
TABLE II. Comparison of the resonance parameters calculated in this work with the ones fitted to the $R$ matrix and experimental cross sections for the $3 s 3 p^{6} n p$ resonances. For each resonance, parameters computed in length gauge are given on the first line, and the ones computed in velocity gauge on the second line.

\begin{tabular}{|c|c|c|c|c|c|c|c|c|c|c|c|c|}
\hline \multirow[b]{2}{*}{$n$} & \multicolumn{4}{|c|}{ This work } & \multicolumn{4}{|c|}{$R$ matrix [29] } & \multirow{2}{*}{$\begin{array}{c}\text { Expt. [38] } \\
\qquad E \\
(\mathrm{eV})\end{array}$} & \multicolumn{3}{|c|}{ Expt. [29] } \\
\hline & $\begin{array}{l}\Delta E \\
(\mathrm{eV})\end{array}$ & $\begin{array}{c}\Gamma \\
(\mathrm{meV})\end{array}$ & $q$ & $\rho^{2}$ & $\begin{array}{c}\Delta E \\
(\mathrm{eV})\end{array}$ & $\begin{array}{c}\Gamma \\
(\mathrm{meV})\end{array}$ & $q$ & $\rho^{2}$ & & $\begin{array}{c}\Gamma \\
(\mathrm{meV})\end{array}$ & $q$ & $\rho^{2}$ \\
\hline \multirow[t]{2}{*}{4} & 26.585 & 87.4 & -0.389 & 0.8391 & 26.633 & 83.8 & -0.383 & 0.843 & 26.605 & $80.2(7)$ & $-0.286(4)$ & $0.840(3)$ \\
\hline & & & -0.442 & 0.8350 & & & -0.433 & 0.846 & & & & \\
\hline \multirow[t]{2}{*}{5} & 27.990 & 29.3 & -0.299 & 0.8452 & 27.997 & 27.4 & -0.292 & 0.825 & 27.994 & $28.5(8)$ & $-0.177(3)$ & $0.848(3)$ \\
\hline & & & -0.345 & 0.8411 & & & -0.342 & 0.829 & & & & \\
\hline \multirow[t]{2}{*}{6} & 28.509 & 13.3 & -0.266 & 0.8467 & 28.508 & 12.4 & -0.262 & 0.824 & 28.509 & $12.2(3)$ & $-0.135(9)$ & $0.852(9)$ \\
\hline & & & -0.310 & 0.8425 & & & -0.312 & 0.827 & & & & \\
\hline \multirow[t]{2}{*}{7} & 28.760 & 7.1 & -0.250 & 0.8475 & 28.756 & 6.7 & -0.249 & 0.823 & 28.757 & $6.6(1)$ & $-0.125(4)$ & $0.846(9)$ \\
\hline & & & -0.293 & 0.8433 & & & -0.299 & 0.827 & & & & \\
\hline \multirow[t]{2}{*}{8} & 28.901 & 4.3 & -0.241 & 0.8476 & 28.896 & 4.0 & -0.240 & 0.824 & 28.898 & $4.5(2)$ & $-0.132(4)$ & $0.77(2)$ \\
\hline & & & -0.284 & 0.8434 & & & -0.291 & 0.827 & & & & \\
\hline \multirow[t]{2}{*}{9} & 28.987 & 2.8 & -0.236 & 0.8477 & 28.928 & 2.6 & -0.235 & 0.823 & & $4.1(2)$ & $-0.115(8)$ & $0.63(3)$ \\
\hline & & & -0.279 & 0.8435 & & & -0.286 & 0.826 & & & & \\
\hline \multirow[t]{2}{*}{10} & 29.045 & 1.9 & -0.233 & 0.8479 & & & & & & & & \\
\hline & & & -0.275 & 0.8436 & & & & & & & & \\
\hline \multirow[t]{2}{*}{11} & 29.085 & 1.3 & -0.230 & 0.8480 & & & & & & & & \\
\hline & & & -0.272 & 0.8437 & & & & & & & & \\
\hline \multirow[t]{2}{*}{12} & 29.114 & 1.0 & -0.228 & 0.8480 & & & & & & & & \\
\hline & & & -0.271 & 0.8438 & & & & & & & & \\
\hline \multirow[t]{2}{*}{13} & 29.135 & 0.7 & -0.227 & 0.8481 & & & & & & & & \\
\hline & & & -0.269 & 0.8438 & & & & & & & & \\
\hline
\end{tabular}

channel accounts for the $10 \%$ discrepancy that can be seen in the lower panel for photon energies above $29.24 \mathrm{eV}$.

We show in Fig. 4 that the window shape of the resonances comes from the dominating $3 p \rightarrow \epsilon d$ channel, while the $3 p \rightarrow \epsilon s$ cross section is instead enhanced on resonance. The partial amplitudes cannot generally be described by the same set of resonance shape parameters. Here $\sigma_{s}$ has, for example, its maximum value at $\epsilon=-q \approx 0.4$, where the total

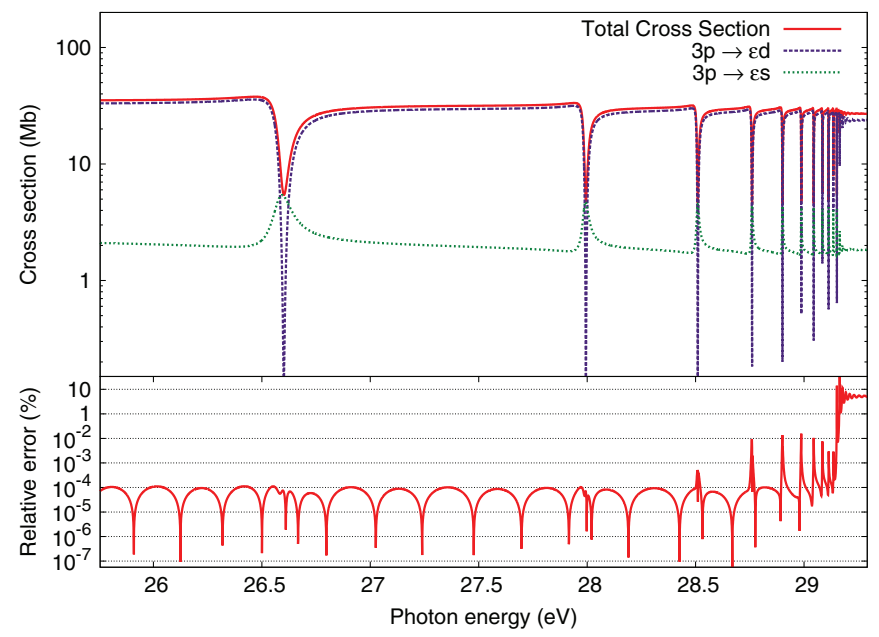

FIG. 4. (Color online) Top panel: partial cross sections for $3 p$ ionization and total cross section, in length gauge. Bottom panel: relative error of the sum of the above partial cross sections with respect to the total cross section computed with Eq. (8), for $R_{0}=65 a_{0}$ and $\theta_{0}=0.055 \mathrm{rad}$. cross section has a minimum. This is due to a destructive interference between $A_{s}^{R}$ and $A_{s}^{D}$ that suppresses the partial background amplitude. This is illustrated in Fig. 5 where the markers correspond to the norm and argument of the calculated partial complex amplitudes in the vicinity of the $3 s 3 p^{6} 4 p$ resonance, while the curves are obtained from the simplified expression

$$
\frac{q+\epsilon}{i+\epsilon}\left|A_{\alpha}^{R}\right|+\eta\left|A_{\alpha}^{D}(0)\right|,
$$

where $\eta=1$ for $\alpha=d$, and $\eta=-1$ for $\alpha=s$. Here, we drop the $3 p^{5}$ label in $\alpha$ for conciseness. The good agreement between the curves and data points illustrates the reliability of the extraction of $A_{\alpha}^{D}(\epsilon)$ and $A_{\alpha}^{R}$ and that $A_{\alpha}^{D}(\epsilon)$ is a slowly varying function. More interestingly, it means that $A_{d}^{D}(\epsilon)$ and $A_{d}^{R}$ are in phase $\left(\arg \left[A_{d}^{D}(\epsilon) / A_{d}^{R}\right] \approx 0\right)$ while $A_{s}^{D}(\epsilon)$ and $A_{s}^{R}$ are in phase opposition $\left(\arg \left[A_{s}^{D}(\epsilon) / A_{s}^{R}\right] \approx \pi\right)$ over a wide range of $\epsilon$. As mentioned in Sec. III D, these relations are rigorously valid if the decay of the bound component of the resonance is treated to lowest order in the Coulomb interaction [34,50]. However, while they hold with increased accuracy as the width of the resonance decreases, they are not exactly realized within more general models like ours.

Another test of our procedure for extracting the $A_{\alpha}^{D}(\epsilon)$ and $A_{\alpha}^{R}$ values, and in particular of $A_{\alpha}(\epsilon)$, is the calculation of $\rho^{2}$. The values from the right-hand side of Eq. (27) agree with those obtained from the total cross section as explained in Sec. II within a couple of parts per thousand. We compare the values obtained from Eq. (27) to the fitted ones from Berrah et al. [29] in Table II. The present results in length gauge agree with experiment up to the $3 s 7 p$ state, while the velocity 

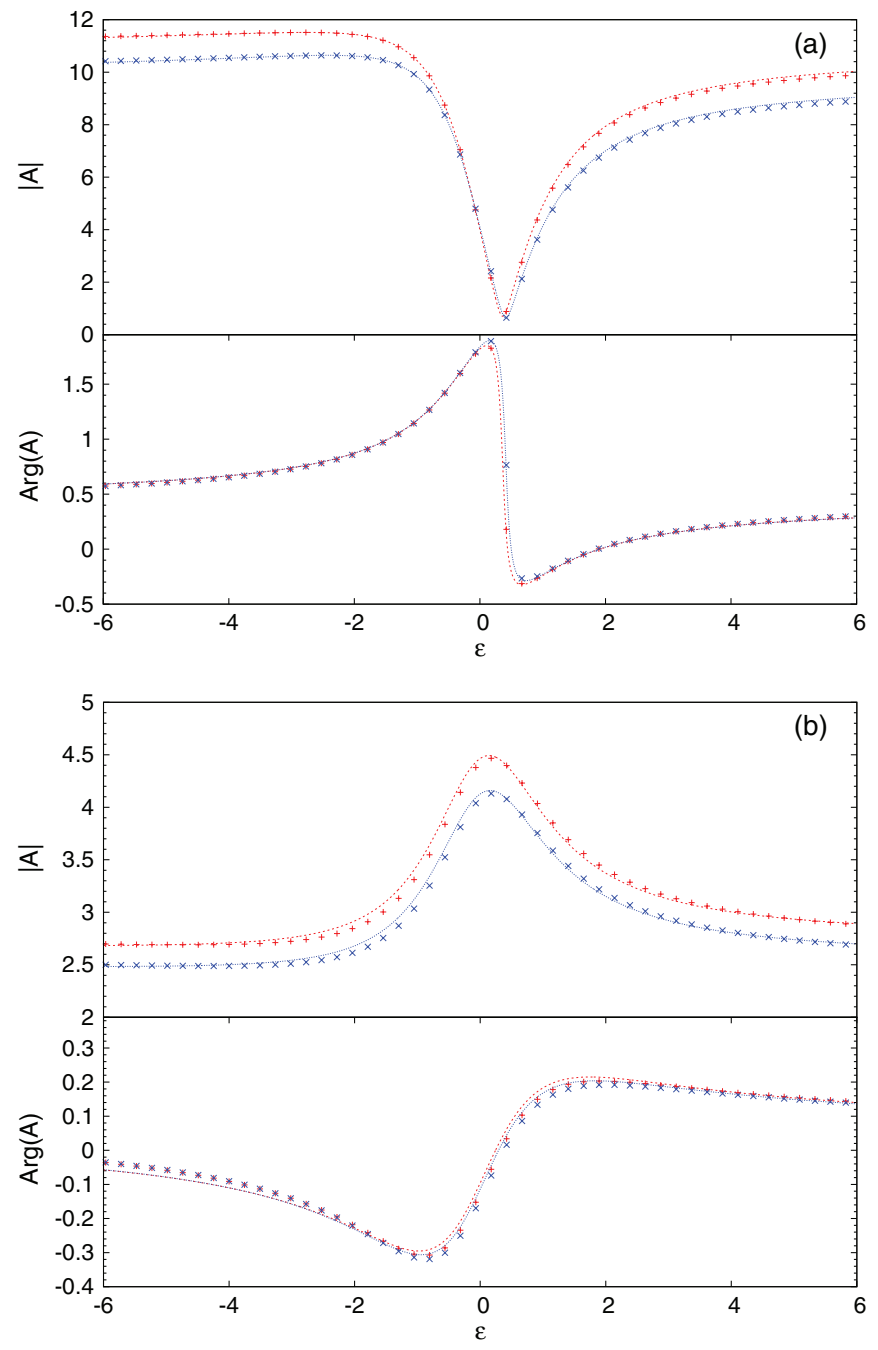

FIG. 5. (Color online) Norm, in atomic units, and argument of the partial photoionization complex amplitudes versus the reduced energy of the $3 s 3 p^{6} 4 p$ resonance. (a) $3 p \rightarrow \epsilon d$ channel. (b) $3 p \rightarrow \epsilon s$ channel. The crosses show 1 on 7 computed values. The lines are obtained from Eq. (29). Red dashed curves and + signs indicate length gauge, and blue full lines and $\times$ signs indicate velocity gauge.

gauge underestimates $\rho^{2}$ slightly. The $R$-matrix fitted values are systematically below ours and the experiment, except in the case of the $3 s 3 p^{6} 4 p$ resonance.

Finally, Fig. 6 compares the calculated $\beta(\omega)$ for the $3 s 3 p^{6} n p$ resonances, $n=4-9$, to the experimental data of Berrah et al. [29]. Overall, the agreement is excellent. No convolution is applied on the theoretical results so that discrepancies appear close to the high $n$ resonances. The $\beta$ parameter is, as expected, about 1.5 where the $d$ channel dominates, far from the resonances (see Fig. 4), while the photoelectron angular distribution is anisotropic $(\beta=0)$ when the $s$ channel dominates, i.e., in the vicinity of the minimum of each Fano structure. The structure in between those two extremes is well described in the calculations, which means that, despite the difference between theory and experiment regarding $q$ values, the relative amplitude and phase between the $s$ and $d$ channels, leading to angular dependent interferences, is well represented.

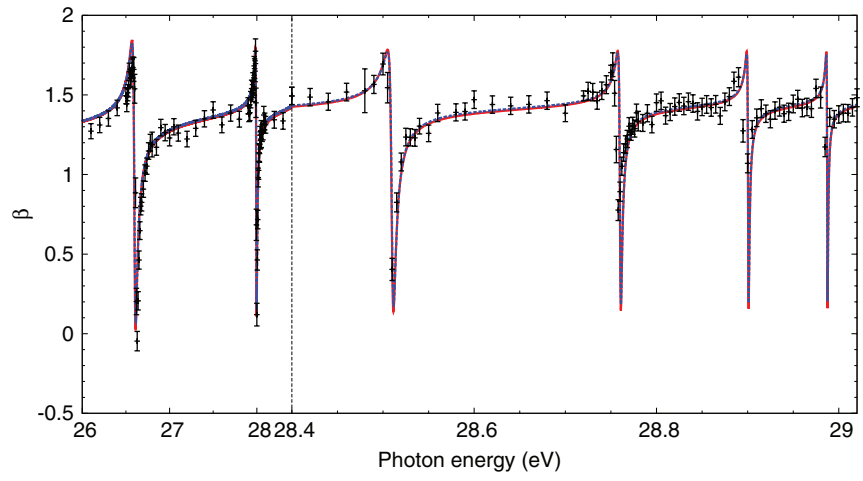

FIG. 6. (Color online) Calculated beta parameter $(\beta)$ in length (full red line) and velocity (broken blue line) compared to experiment [29] (black dots). The vertical dashed line indicates a change of photon energy scale.

\section{APPLICATION TO PHOTOIONIZATION DELAY MEASUREMENTS}

Photoionization by attosecond XUV pulses assisted by phase-locked IR laser-probe fields is a widely used experimental scheme to gain temporal information about subfemtosecond pulse structures and dynamics [54]. This laser-assisted photoionization process is often interpreted as an attosecond streak-camera method, where the photoelectron will gain momentum from the instantaneous vector potential of the probe field depending on its exact time of ionization by the attosecond pulse. Assuming that the intensity of both pulses is weak, the corresponding light-matter interaction can be treated using lowest-order perturbation theory and the onset of this streaking mechanism can be described with high fidelity using only a limited number of complex transition amplitudes [55]. The delay-dependent probability modulations of the photoelectron distributions are then directly related to the phase differences of the two-photon, two-color matrix elements (XUV $\pm \mathrm{IR})$. These so-called atomic phases in laser-assisted photoionization [56], have been implemented for reliable characterization schemes of attosecond pulses [57]. Recently, similar experimental techniques have been extended to study the delay in photoionization from different initial states in neon [7] and argon atoms [8,9]. Theoretical work has shown that the total atomic delay, $\tau_{A}$, measured in these experiments can be approximated as the sum of the one-photon Wigner-like delay in photoionization, $\tau_{W}$, plus the so-called continuum-continuum delay, $\tau_{c c}$, which is induced by the laserprobe field and the long-range ionic potential, so that $[8,55,58]$

$$
\tau_{A}=\tau_{W}+\tau_{\mathrm{cc}}
$$

In order to evaluate $\tau_{W}$, we first apply the method presented in Sec. III C in order to extract the partial wave amplitudes, $A_{\alpha}(\omega)$, which contain the asymptotic phase shifts, $\delta_{\alpha}(\omega) \equiv \arg \left[A_{\alpha}(\omega)\right]$, relative to the Coulombic phase shifts, $\sigma_{l}(\omega)$, where $l$ is the angular momentum of the photoelectron in channel $\alpha$. Then, using the total asymptotic phase shift, $\eta_{\alpha}(\omega) \equiv \sigma_{l}(\omega)+\delta_{\alpha}(\omega)$, we construct the Wigner-like delay for channel $\alpha$ as a finite-difference derivative

$$
\tau_{W}^{(\alpha)} \equiv \frac{\eta_{\alpha}\left(\omega_{>}\right)-\eta_{\alpha}\left(\omega_{<}\right)}{2 \omega_{\mathrm{IR}}}
$$


where the difference between the XUV frequencies that will interfere due to the influence of the probe field are separated by two IR probe photons, $\omega_{>}-\omega_{<}=2 \omega_{\mathrm{IR}}$.

The absorption of a laser-probe photon, required to make the transition from the intermediate momentum $k_{i}$ to the final momentum $k_{f}$, induces a phase shift denoted $\phi_{\mathrm{cc}}$. Starting from the formalism presented in Ref. [10], we use the soft-photon approximation $k_{f}^{2} / 2 \gg \omega_{\mathrm{IR}}$ and find the following expression for the phase shift, using atomic units $\left(\hbar=1, e=1, m_{e}=1\right.$, and $4 \pi \epsilon_{0}=1$ ):

$$
\phi_{\mathrm{cc}}\left(k_{f}, \omega_{\mathrm{IR}}\right)=\arg \left\{\left(\frac{k_{f}}{i \omega_{\mathrm{IR}}}\right)^{i \omega_{\mathrm{IR}} Z / k_{f}^{3}} \Gamma\left[1+\frac{i \omega_{\mathrm{IR}} Z}{k_{f}^{3}}\right]\right\},
$$

where $Z=1$ is the charge of the target ion. Equation (32) is accurate for photoelectrons with high kinetic energy. The continuum-continuum delay can then be written as

$$
\tau_{\mathrm{cc}}\left(k_{f}, \omega_{\mathrm{IR}}\right) \approx-\frac{\phi_{\mathrm{cc}}\left(k_{f}, \omega_{\mathrm{IR}}\right)}{\omega_{\mathrm{IR}}} .
$$

Alternatively this delay could be estimated with other methods such as the eikonal Volkov approximation [59] or classicaltrajectory Monte Carlo simulations [58].

If there is more than one channel contributing to a given final state, the two-photon amplitudes have to be added in a consistent way depending on the experimental observable [56]. Such a detailed analysis is beyond the scope of this paper, where we will show that the correlated Wigner-like delay Eq. (31) from the dominant channels is enough to explain the observations within the experimental uncertainty.

Klünder et al. [8] and Guénot et al. [9] have measured the $3 s-3 p$ atomic delay difference at XUV photon energies of $34.1,37.2$, and $40.3 \mathrm{eV}$ corresponding to the high-order harmonic sidebands 22,24 , and 26 for a generating field of $1.55 \mathrm{eV}$. The contribution from the continuum-continuum delay was then subtracted according to Eq. (30), in order to isolate the Wigner-like delay in photoionization.

The first pseudoresonance in our model lies at an energy of $39.4 \mathrm{eV}$, which is inside the range of the experimental measurement, leading to an unphysical feature at $37.8 \mathrm{eV}$. In order to assess the reliability of the computed delays, we use a reduced close-coupling expansion by excluding the $N$ and $(N+1)$-electron CSF involving the $4 s$ correlation orbital, which is responsible for this artifact. In this truncated model, the energy scale is shifted downward by $0.45 \mathrm{eV}$ to match the Ar $3 s$ photoemission threshold. Another pseudoresonance remains at $41.3 \mathrm{eV}$, affecting the Wigner-like delay at energies close to the sideband 26 , preventing comparison with the last experimental value.

In Fig. 7(a) we present the Wigner-like delay obtained with the reduced model. It is computed by Eq. (31) for channels leading to photoemission from the $3 p$ and $3 s$ orbitals. In Fig. 7(b) we show the delay difference between the two dominant channels, $3 s 3 p^{6} \epsilon p$ and $3 p^{5} \epsilon d$, using the full and reduced close-coupling models. An exact determination of $\tau_{\mathrm{cc}}$ is difficult and we, therefore, compare these curves to the original experimental data points for the Wigner-like delay of Ref. [9] together with the ones estimated with the alternative $\tau_{\mathrm{cc}}$ from Eq. (33) and Ref. [59]. There is good agreement

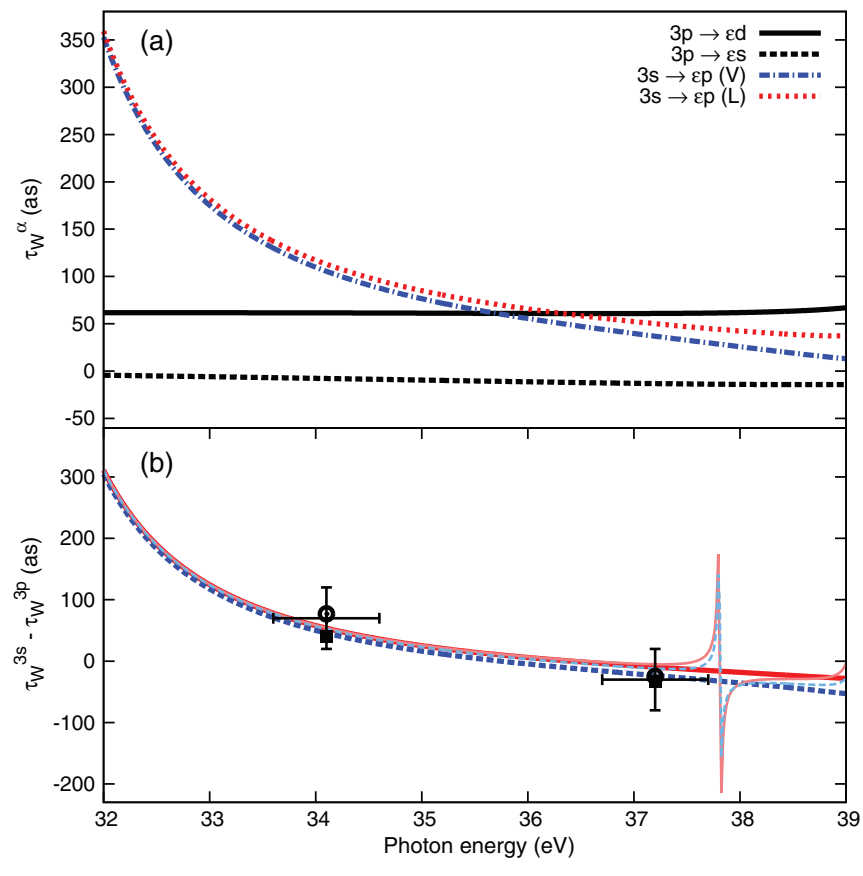

FIG. 7. (Color online) (a) Wigner-like delays for photoionization from argon. There is no perceptible gauge dependence for photoionization from the $3 p$ orbital. For the $3 s$ photoionization, the results from velocity (blue broken) and length gauge (red dashed) are shown. (b) Wigner-like delay difference between photoionization from the $3 p$ and $3 s$ orbitals in argon. The thin curves are obtained with the full model that exhibit a pseudoresonance at $39.4 \mathrm{eV}$. The thick curves are obtained with the truncated model for velocity (blue dashed) and length (red full) gauge, respectively. The experimental values of Ref. [9] are given with error bars. The experimental data point obtained using the $\tau_{\mathrm{cc}}$ from Eq. (33) and Ref. [59] are given by the circles and squares, respectively.

between our theoretical model and the experimental points of sidebands 22 and 24.

Going beyond the present analysis requires one to include the many shake-up thresholds opening at these energies [52], as shown in Fig. 1. As seen in experimental data [60] and in $R$-matrix calculations [61], the $3 s$ photoionization partial and the total cross sections are highly structured by resonances associated to those thresholds. This suggests that the corresponding phases may be strongly altered as well. However, the widths of these resonances are in general below $0.1 \mathrm{eV}$ so that we can expect that they do not drastically affect the measured delays obtained from an average over the sidebands. Still, as we will show below, some structure should be observable provided that the IR probe field is sufficiently narrow.

The sideband amplitude can be obtained as the convolution of the IR spectral envelope with the dipole element between the perturbed wave function and the final state [10]. If, as before, we limit our discussion to Eq. (30), we can estimate the effect of the probe field bandwidth $\Gamma_{\mathrm{IR}}$ by extracting the Wigner-like delay from the complex amplitudes convoluted with a Gaussian function

$$
\tilde{A}_{\alpha}(\omega)=\sqrt{\frac{4 \ln 2}{\pi \Gamma_{\mathrm{IR}}^{2}}} \int d x A_{\alpha}(x) \exp \left[-\frac{4 \ln 2(\omega-x)^{2}}{\Gamma_{\mathrm{IR}}^{2}}\right] .
$$




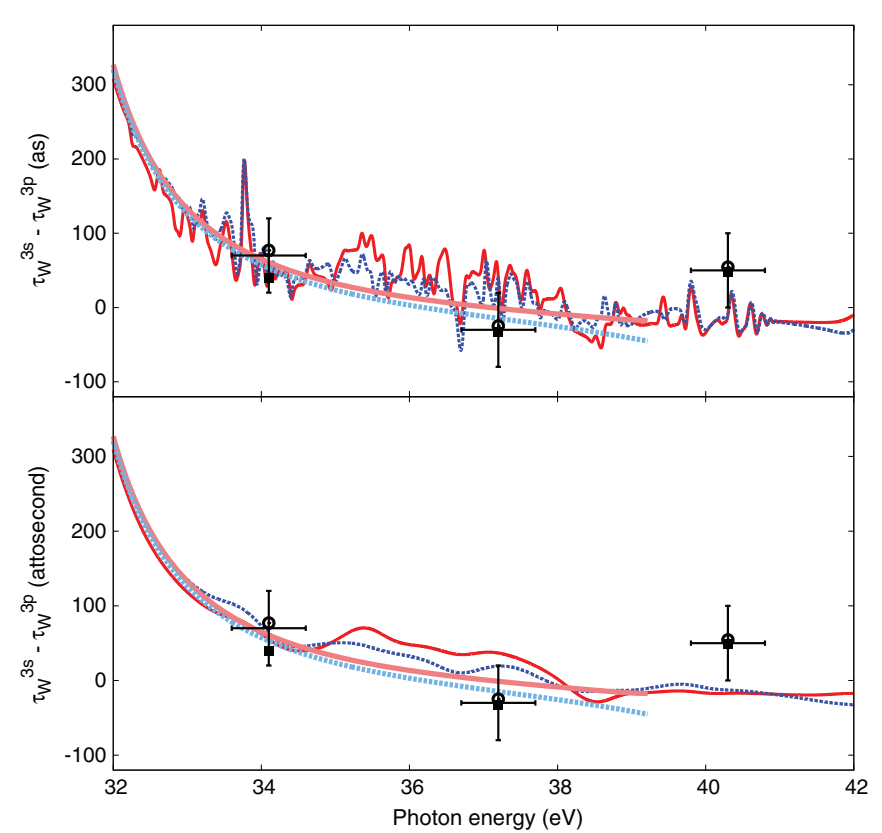

FIG. 8. (Color online) Relative Wigner-like delays using a model including some doubly excited resonances (see the Appendix). (a) The probe field bandwidth is $60 \mathrm{meV}$ and $0.6 \mathrm{eV}$, respectively, for the top and bottom panels. Length (continuous red) and velocity (dashed blue) gauge results are shown. The thicker, light curves correspond to the reduced model used in Fig. 7. Experimental points are shown with the same notation as in Fig. 7.

In Fig. 8, we present Wigner-like delay differences obtained with a model that includes a range of physical resonances associated to the thresholds in black in Fig. 1 (details are found in the Appendix), after smoothing by the bandwidth of the probe field using Eq. (34). The top panel shows the results for a Gaussian IR envelope with FWHM of $60 \mathrm{meV}$, corresponding to an IR field duration of $30 \mathrm{fs}$, typical of experiments using trains of attosecond pulses. The bottom panel corresponds to a FWHM of $0.6 \mathrm{eV}$, corresponding to a single IR cycle probe pulse, typical of experiments using isolated attosecond pulses. We show that the presence of resonances does not radically change the baseline of the relative delays, but rather superimposes a structure that preserves the previous theory-experiment agreement. Including the physical resonances in the model also pushes pseudoresonances higher in the spectrum so that a comparison can be made with the experimental delay extracted at the sideband 26. Here, some limited agreement can still be found in the top panel, within the theoretical and experimental uncertainty. The Cooper minimum emphasized in Ref. [9], experimentally located at about $43 \mathrm{eV}$, is also located at higher photon energies in our calculations so that it does not affect the computed delays. We should also note that, above $39 \mathrm{eV}$, many more resonances could affect $\tau_{W}^{\alpha}$, corresponding to shake-up electrons in higher orbitals, even though they seem to be less important as far as the cross section is concerned [60].

An important implication of these results is that a rich structure, originating from resonances, could be observed experimentally by scanning the energy of the high-order harmonics [see Fig. 8(a)]. In order to observe such features, the bandwidth of the IR field should be sufficiently narrow. Otherwise, if a too short probe pulse is used, which is often the case in attosecond streaking experiments, these sharp energy-dependent features cannot be resolved. In this case, the resonances may affect the measured delay smoothly, as shown in Fig. 8(b), where they cause an additional delay of up to several tens of attoseconds as compared to the model without resonances in this energy region.

Such a streaking experiment has been performed in neon to extract a relative photoionization delay of 21(5) as between the $2 p$ and $2 s$ electron extraction [7]. No theoretical analysis has been able to reproduce this result so far. Two of them include the effects of a probe field within an ab initio many-body framework. Full-fledged simulations of the measurement have been performed by Moore et al. [62], using time-dependent $R$-matrix theory, resulting in a relative delay of 10.2(1.3) as Later, we have reproduced this result using two-photon matrix elements computed using many-body perturbation theory [63]. In that study, the validity of Eq. (30) is demonstrated. In both theoretical approaches the effects of transient double excitations are neglected. As in the case of argon, the $\mathrm{Ne}^{+}$ level structure at the relevant energy, i.e., an XUV energy of about $105 \mathrm{eV}$, is extremely rich [52]. Here we have seen that such effects might play an important role. In neon, an extra complication is that the experimental photon energy is above several double ionization thresholds, and that triply excited states may also be important, as pointed out in Ref. [62]. This implies that it will be important to include a complete description of at least two active electrons, and possibly excited configurations of the grandparent system.

\section{CONCLUSIONS AND OUTLOOK}

We have presented a practical method, based on the Atomic Structure Package (ATSP2K) [33], to obtain a general, manyelectron atomic wave function in a close-coupling expansion. The structure of the localized part of the wave function is represented using the multiconfiguration Hartree-Fock (MCHF) method, which provides a versatile and flexible way to represent complex many-body effects with a limited number of configurations. By using $B$-splines to describe the radial components of the photoelectron in the allowed partial-wave channels, we obtain a multichannel method that, for the description of one active electron in a box, is complete. This paper develops this approach in combination with the exterior complex scaling method of use, in particular, in the description of monochromatic wave packets. In addition to evaluating the total cross section with the standard tools of complex scaling, we have extracted complex partial amplitudes that encode the full information on the studied process. The consistency between the two approaches has been verified in detail.

The method is validated by application to the photoionization of argon close to the $3 s$ ionization threshold. In this energy region the final state is affected by strong configuration interaction. We found good agreement of the line profiles of the $3 s 3 p^{6} n p$ autoionizing states with previous $R$-matrix calculations and experiments. Also the computed photoelectron angular distribution is in excellent agreement with the measurements.

It has earlier been suggested that laser-assisted photoionization by attosecond pulses gives access, in a universal way, to relative one-photon Wigner-like delays (see, e.g., 
Refs. [7-9]). So far, this interpretation of the observations has remained uncertain since no $a b$ initio calculations have been able to reproduce the experimental data, even though some encouraging results were obtained in the case of argon $[9,63]$. In this paper, we present a study that contributes to validation of these claims, in the context of a weak probe field, by including all relevant correlation effects, and by showing that, in so doing, satisfactory agreement is obtained with the observed atomic delay shifted by the atom-independent continuum-continuum delay. We also show that the presence of many resonances decaying into $\operatorname{Ar}^{+}\left(3 p^{5}\right)$ and $\mathrm{Ar}^{+}\left(3 s 3 p^{6}\right)$ in the studied energy region affects the result of the measurements in ways that are sensitive to experimental parameters. In a future study we will extend the treatment to include consistent $a b$ initio calculations of the single photon and laser-assisted photoelectron emission delays, in a similar way as done in Ref. [63].

However, it has been shown for the helium atom that dynamical screening effects caused by the polarization of the parent ion by the IR field can affect the delay of electron emission [64]. The rich structure of the $\mathrm{Ar}^{+}$spectrum at the relevant energies [52] suggests that such an effect could be significant in the present case. A straightforward possibility to include those effects in the calculation is to move to a time-dependent approach, including explicitly the shape of the IR pulse in order to account for the full many-body dynamics of the process, both on the relatively long time scale of autoionization, and the subfemtosecond scale set by the XUV pulse.

For further developments we note that the compactness of the MCHF and $B$-spline close-coupling approach presented here makes it well suited for time-dependent many-body calculations on systems in which exact calculations are untractable. Only a limited number of such calculations exist today (see, for example, [62,65-68]), all of them performed within the time-dependent $R$-matrix framework $[67,69,70]$. As a next step we will combine the present approach with a solution of the time-dependent Schrödinger equation along the lines of Refs. [71,72], where the calculation is made without complex scaling and the final physical results are extracted by projection of the finite-time wave packet on $K$-matrix scattering states. Absorbing boundaries are then used in order to avoid reflections on the walls of the computational box in the case of long simulations. We foresee here that the common description of the structure part in the time-independent and time-dependent calculations will be of significant value for the tuning of the approximations regarding many-body effects that has to be introduced in the time-dependent approach.

\section{ACKNOWLEDGMENTS}

We thank Professor Nora Berrah and Dr. Burkhard Langer for providing us with experimental data. Financial support from the Swedish Research Council (VR) and the WennerGren Foundation is gratefully acknowledged. L.A. acknowledges funding from the European Research Council under the European Union's Seventh Framework Program (FP7/20072013)/ERC grant agreement No. 290853, and the MICINN No. FIS2010-15127. We are grateful for financial support from the European COST Action CM0702.
TABLE III. Comparison of the lowest computed levels of $\mathrm{Ar}^{+}$to $R$-matrix calculations [61] and experimental data [73].

\begin{tabular}{lccc}
\hline \hline State & This work & Ref. [61] & Expt. [73] \\
\hline $3 p^{5}{ }^{2} P^{o}$ & 16.481 & 16.303 & 15.819 \\
$3 s 3 p^{6}{ }^{2} S^{e}$ & 29.24 & 29.24 & 29.24 \\
$3 p^{4}\left({ }^{1} D\right) 4 s^{2} D^{e}$ & 34.699 & 34.999 & 34.203 \\
$3 p^{4}\left({ }^{3} P\right) 3 d^{2} D^{e}$ & 35.418 & 35.427 & 34.462 \\
$3 p^{4}\left({ }^{3} P\right) 4 p^{2} P^{o}$ & 36.044 & 36.514 & 35.605 \\
$3 p^{4}\left({ }^{1} S\right) 4 s^{2} S^{e}$ & 37.394 & 37.207 & 36.504 \\
$3 p^{4}\left({ }^{1} D\right) 4 p^{2} P^{o}$ & 37.654 & 38.134 & 37.137 \\
$3 p^{4}\left({ }^{1} D\right) 3 d^{2} D^{e}$ & 38.647 & 38.508 & 37.148 \\
$3 p^{4}\left({ }^{1} S\right) 3 d^{2} D^{e}$ & 39.498 & 39.296 & 38.043 \\
$3 p^{4}\left({ }^{1} D\right) 3 d^{2} S^{e}$ & 39.595 & 39.876 & 38.585 \\
\hline \hline
\end{tabular}

\section{APPENDIX}

Here, we give the details of the calculations used to produce Fig. 8. The model is aimed at a qualitative description of the spectral features in the range of the experiments performed in Refs. [8,9].

According to Ref. [61], the most prominent features in the $3 s$ photoionization partial cross section and total cross section, come from the resonances of the type $3 p^{4} n l[L S \pi] n^{\prime} l^{\prime}$ with $n l=3 d, 4 s$, and $4 p, n^{\prime} \leqslant 7$, and for the parent term $L S \pi={ }^{2} S^{e},{ }^{2} P^{o},{ }^{2} P^{e}$, and ${ }^{2} D^{e}$. The ${ }^{2} P^{e}$ parent states are mostly responsible for resonances at $31 \mathrm{eV}$ and below so we do not consider them. Using the same $1 s, 2 s, 2 p, 3 s$, and $3 p$ orbitals as before, we perform Hartree-Fock level average calculations on the $3 p^{4} n l$ states optimizing the $3 d, 4 s$, and $4 p$ orbitals. Extending the active set of spectroscopic orbitals by a $\overline{4 d}$ correlation orbital, we perform a MCHF calculation on the lowest state of the ${ }^{2} S^{e}$ symmetry of $\mathrm{Ar}^{+}$including all single and double (SD) excitations of the multireference set $(\mathrm{MR})\left\{3 s 3 p^{6} \cup 3 p^{4} 4 s \cup 3 p^{4} 3 d \cup 3 p^{4} \overline{4 d}\right\}$. Configuration interaction calculations are then performed on the ${ }^{2} P^{o}$ and ${ }^{2} D^{e}$ states following the same MR-SD scheme with the references $\left\{3 p^{4} 4 s \cup 3 p^{4} 3 d \cup 3 p^{4} \overline{4 d}\right\}$ and $\left\{3 p^{5} \cup 3 p^{4} 4 p\right\}$, respectively. Then, the CSF with a mixing coefficient over 0.07 are selected to build the target states. The lowest levels of $\mathrm{Ar}^{+}$obtained in this model are compared to experiment in Table III. Most values agree with the experiment within $1 \mathrm{eV}$.

The localized states $\chi_{\xi}$ are chosen such that the closecoupling expansion Eq. (5) still retains the completeness of the $B$-spline set. Using this model, the photon energy scale is shifted by $0.15 \mathrm{eV}$ upward in order to match the experimental $3 s$ photoionization. We choose a knot sequence as before for

TABLE IV. Computed Fano profile parameters for the for the $3 s 3 p^{6} n p$ resonances. Positions are shifted upward by $0.15 \mathrm{eV}$.

\begin{tabular}{|c|c|c|c|c|}
\hline$n$ & $\begin{array}{c}\Delta E \\
(\mathrm{eV})\end{array}$ & $\begin{array}{c}\Gamma \\
(\mathrm{meV})\end{array}$ & $q(\mathrm{~L})$ & $q(\mathrm{~V})$ \\
\hline 4 & 26.524 & 81.0 & -0.497 & -0.646 \\
\hline 5 & 28.005 & 25.0 & -0.435 & -0.489 \\
\hline 6 & 28.516 & 11.7 & -0.409 & -0.443 \\
\hline 7 & 28.764 & 6.4 & -0.396 & -0.423 \\
\hline 8 & 28.903 & 3.9 & -0.389 & -0.412 \\
\hline
\end{tabular}


the inner region up to $1 / Z a_{0}$, but with a linear step of $1 a_{0}$ up to $51 a_{0}$ and then an exponentially increasing step up to $150 a_{0}$ for accommodating orbitals with $n \leqslant 7$. The complex scaling starts at $40 a_{0}$ with a complex scaling angle of $0.11 \mathrm{rad}$. As in Sec. IV, there is only a negligible difference between the sum of the partial cross sections and the total cross section computed with Eq. (7). In Table IV, we provide the results obtained for the $3 s 3 p^{6} n p$ resonance line shapes. A good consistency is found with the values given in Table II, except for the $q$ parameters. Furthermore, the partial cross sections obtained within this model exhibit strong features in qualitative agreement with $R$-matrix calculations [61].
[1] N. R. Badnell, M. A. Bautista, K. Butler, F. Delahaye, C. Mendoza, P. Palmeri, C. J. Zeippen, and M. J. Seaton, Mon. Not. R. Astron. Soc. 360, 458 (2005).

[2] B. Boudaïffa, P. Cloutier, D. Hunting, M. A. Huels, and L. Sanche, Science 287, 1658 (2000).

[3] U. Fano and J. W. Cooper, Rev. Mod. Phys. 40, 441 (1968).

[4] A. F. Starace, Appl. Opt. 19, 4051 (1980).

[5] G. Sansone, L. Poletto, and M. Nisoli, Nat. Photonics 5, 655 (2011).

[6] L. Young, E. P. Kanter, B. Krässig, Y. Li, A. M. March, S. T. Pratt, R. Santra, S. H. Southworth, N. Rohringer, L. F. DiMauro et al., Nature (London) 466, 053001 (2010).

[7] M. Schultze, M. Fiess, N. Karpowicz, J. Gagnon, M. Korbman, M. Hofstetter, S. Neppl, A. L. Cavalieri, Y. Komninos, T. Mercouris et al., Science 328, 1658 (2010).

[8] K. Klünder, J. M. Dahlström, M. Gisselbrecht, T. Fordell, M. Swoboda, D. Guénot, P. Johnsson, J. Caillat, J. Mauritsson, A. Maquet et al., Phys. Rev. Lett. 106, 143002 (2011).

[9] D. Guénot, K. Klünder, C. L. Arnold, D. Kroon, J. M. Dahlström, M. Miranda, T. Fordell, M. Gisselbrecht, P. Johnsson, J. Mauritsson et al., Phys. Rev. A 85, 053424 (2012).

[10] J. M. Dahlström, A. L'Huillier, and A. Maquet, J. Phys. B 45, 183001 (2012).

[11] P. G. Burke and K. T. Taylor, J. Phys. B 8, 2620 (1975).

[12] L. Moore, M. Lysaght, L. Nikolopoulos, J. Parker, H. van der Hart, and K. Taylor, J. Mod. Opt. 58, 1132 (2011).

[13] R. Moccia and P. Spizzo, Phys. Rev. A 43, 2199 (1991).

[14] L. Argenti and R. Moccia, J. Phys. B 43, 235006 (2010).

[15] T. N. Rescigno and V. McKoy, Phys. Rev. A 12, 522 (1975).

[16] E. Lindroth, Phys. Rev. A 52, 2737 (1995).

[17] K. T. Chung, Phys. Rev. Lett. 78, 1416 (1997).

[18] B. Simon, Phys. Lett. A 71, 211 (1979).

[19] C. W. McCurdy, T. N. Rescigno, and D. Byrum, Phys. Rev. A 56, 1958 (1997).

[20] T. N. Rescigno, M. Baertschy, D. Byrum, and C. W. McCurdy, Phys. Rev. A 55, 4253 (1997).

[21] D. A. Horner, F. Morales, T. N. Rescigno, F. Martín, and C. W. McCurdy, Phys. Rev. A 76, 030701 (2007).

[22] T. N. Rescigno, W. Vanrose, D. A. Horner, F. Martín, and C. W. McCurdy, J. Electron Spectrosc. Relat. Phenom. 161, 85 (2007).

[23] C. W. McCurdy, M. Baertschy, and T. N. Rescigno, J. Phys. B 37, R137 (2004)

[24] I. Lindgren and J. Morrison, Atomic Many-Body Theory, Series on Atoms and Plasmas, 2nd ed. (Springer-Verlag, New York, 1986).

[25] C. Froese Fischer, T. Brage, and P. Jönsson, Computational Atomic Structure: An MCHF Approach, 1st ed. (Taylor \& Francis, London, 1997).

[26] W. R. Johnson, Atomic Structure Theory: Lectures on Atomic Physics, 1st ed. (Springer-Verlag, New York, 2007).
[27] C. Froese Fischer, Hartree-Fock Methodfor Atoms: A Numerical Approach (Wiley, New York, 1977).

[28] C. Froese Fischer and P. Jönsson, Comput. Phys. Commun. 84, 37 (1994).

[29] N. Berrah, B. Langer, J. Bozek, T. W. Gorczyca, O. Hemmers, D. W. Lindle, and O. Toader, J. Phys. B 29, 5351 (1996).

[30] D.-S. Kim, H.-L. Zhou, and S. T. Manson, Phys. Rev. A 55, 414 (1997).

[31] H. Massey and C. Mohr, Proc. R. Soc. London, Ser. A 136, 289 (1932).

[32] H. Massey and C. Mohr, Proc. R. Soc. London, Ser. A 139, 187 (1933).

[33] C. Froese Fischer, G. Tachiev, G. Gaigalas, and M. R. Godefroid, Comput. Phys. Commun. 176, 559 (2007).

[34] N. M. Kabachnik and I. P. Sazhina, J. Phys. B 9, 1681 (1976).

[35] M. Amusia and A. S. Kheifets, Phys. Lett. A 89, 437 (1982).

[36] S. L. Sorensen, T. Åberg, J. Tulkki, E. Rachlew-Källne, G. Sundström, and M. Kirm, Phys. Rev. A 50, 1218 (1994).

[37] R. P. Madden, D. L. Ederer, and K. Codling, Phys. Rev. 177, 136 (1969).

[38] S. L. Wu, Z. P. Zhong, R. F. Feng, S. L. Xing, B. X. Yang, and K. Z. Xu, Phys. Rev. A 51, 4494 (1995).

[39] K. T. Taylor, J. Phys. B 10, L699 (1977).

[40] K. Codling, J. B. West, A. C. Parr, J. L. Dehmer, and R. L. Stockbauer, J. Phys. B 13, L693 (1980).

[41] A. Svensson, M. O. Krause, and T. A. Carlson, J. Phys. B 20, L271 (1987).

[42] A. Kramida, Y. Ralchenko, J. Reader, and N. A. Team, NIST Atomic Spectra Database (version 5.0), 2012, http://physics.nist.gov/asd.

[43] C. deBoor, A Practical Guide to Splines (Springer-Verlag, New York, 1978).

[44] C. W. McCurdy, D. A. Horner, T. N. Rescigno, and F. Martín, Phys. Rev. A 69, 032707 (2004).

[45] J. E. Hansen, M. Bentley, H. W. van der Hart, M. Landtman, G. M. S. Lister, Y.-T. Shen, and N. Vaeck, Phys. Scr. T 47, 7 (1993).

[46] L. Argenti and R. Colle, Comput. Phys. Commun. 180, 1442 (2009).

[47] O. Zatsarinny, Comput. Phys. Commun. 174, 273 (2006).

[48] J. Bengtsson, E. Lindroth, and S. Selst $\varnothing$, Phys. Rev. A 78, 032502 (2008).

[49] U. Fano, Phys. Rev. 124, 1866 (1961).

[50] U. Fano and J. W. Cooper, Phys. Rev. 137, A1364 (1965).

[51] R. Sternheimer, Phys. Rev. 84, 244 (1951).

[52] A. Kikas, S. J. Osborne, A. Ausmees, S. Svensson, O.-P. P. Sairanen, and S. Aksela, J. Electron. Spectrosc. Relat. Phenom. 77, 241 (1996).

[53] J. Samson and W. Stolte, J. Electron Spectrosc. Relat. Phenom. 123, 265 (2002). 
[54] F. Krausz and M. Ivanov, Rev. Mod. Phys. 81, 163 (2009).

[55] J. M. Dahlström, D. Guénot, K. Klünder, M. Gisselbrecht, J. Mauritsson, A. L'Huillier, A. Maquet, and R. Taïeb, Chem. Phys. (2012), doi: 10.1016/j.chemphys.2012.01.017.

[56] E. S. Toma and H. G. Muller, J. Phys. B 35, 3435 (2002).

[57] P. M. Paul, E. S. Toma, P. Breger, G. Mullot, F. Augé, P. Balcou, H. G. Muller, and P. Agostini, Science 292, 1689 (2001).

[58] S. Nagele, R. Pazourek, J. Feist, K. Doblhoff-Dier, C. Lemell, K. Tőkési, and J. Burgdörfer, J. Phys. B 44, 081001 (2011).

[59] M. Ivanov and O. Smirnova, Phys. Rev. Lett. 107, 213605 (2011).

[60] B. Möbus, B. Magel, K.-H. Schartner, B. Langer, U. Becker, M. Wildberger, and H. Schmoranzer, Phys. Rev. A 47, 3888 (1993).

[61] H. W. van der Hart and C. H. Greene, Phys. Rev. A 58, 2097 (1998).

[62] L. R. Moore, M. A. Lysaght, J. S. Parker, H. W. van der Hart, and K. T. Taylor, Phys. Rev. A 84, 061404 (2011).
[63] J. M. Dahlström, T. Carette, and E. Lindroth, Phys. Rev. A 86, 061402 (2012).

[64] R. Pazourek, J. Feist, S. Nagele, and J. Burgdörfer, Phys. Rev. Lett. 108, 163001 (2012).

[65] H. W. van der Hart, M. A. Lysaght, and P. G. Burke, Phys. Rev. A 76, 043405 (2007)

[66] X. Guan, C. J. Noble, O. Zatsarinny, K. Bartschat, and B. I. Schneider, Phys. Rev. A 78, 053402 (2008).

[67] M. A. Lysaght, H. W. van der Hart, and P. G. Burke, Phys. Rev. A 79, 053411 (2009).

[68] S. Hutchinson, M. A. Lysaght, and H. W. van der Hart, J. Phys. B 44, 215602 (2011).

[69] P. G. Burke and V. M. Burke, J. Phys. B 30, L383 (1997).

[70] X. Guan, O. Zatsarinny, K. Bartschat, B. I. Schneider, J. Feist, and C. J. Noble, Phys. Rev. A 76, 053411 (2007).

[71] L. Argenti and E. Lindroth, Phys. Rev. Lett. 105, 053002 (2010).

[72] L. Argenti, R. Pazourek, J. Feist, S. Nagele, M. Liertzer, E. Persson, J. Burgdörfer, and E. Lindroth, arXiv:1210.2187.

[73] L. Minnhagen, Ark. Fys. 25, 203 (1963). 